\section{Posterior Cerebral Artery}

Elliot J. Roth

Northwestern University, Feinberg School of Medicine, Physical Medicine and Rehabilitation, Chicago, IL, USA

\section{Synonyms}

Cerebral artery

\section{Definition}

The two posterior cerebral arteries supply blood to the posterior aspect of the brain, particularly the occipital lobe. They form as the terminal branches at the bifurcation of the ascending basilar artery. The posterior cerebral artery gives off several branches that supply blood to the brain stem and thalamus, deeper portions of the brain, occipital lobe, and part of the cerebellum. Each posterior cerebral artery also gives rise to a posterior communicating artery, which connects the posterior cerebral circulation to the internal carotid artery and the anterior cerebral circulation.

Occlusion of the posterior circulation can cause various brainstem syndromes, loss of sensation, visual field defects, cranial nerve palsies, tremor, cognitive and perceptual deficits, hallucinations, and other findings, depending on the specific location of the lesion.

\section{Cross-References}

- Basilar Artery

- Brainstem Stroke

- Circle of Willis

- Posterior Communicating Artery

- Vertebrobasilar System 